\title{
La confusion du juste chez Épicure, Hermarque et Lucrèce. Une inégalité intellectuelle des citoyenes?
}

\author{
Justice confused in Epicurus, Hermarch and Lucretius. \\ An intellectual inequality of citizens?
}

\author{
JULIE GIOVACGHINI \\ CNRS.ENS-PSL \\ julie.giovacchini@cnrs.fr
}

Recibido: 30/09/2019 - Aceptado: 23/09/2020
DOI https://doi.org/10.20318/fons.2020.5052

\begin{abstract}
This article aims to confront a serious difficulty in Epicurean political thought, through the investigation of a likely contradiction between the universal and soteriological ambition of Epicureanism, which presents itself as wisdom addressed to all and adapted to all, and the portrayal of a humanity with highly diverse intellectual capacities; within this humanity some individuals would not be able to attain an active and autonomous political consciousness. Despite the weakness of the textual sources, some elements of this political thought are known thanks to fragments of Hermarchus' Against Empedocles, which develop some of the lapidary hypotheses outlined by Epicurus in the last Kuriai Doxai. A particularly interesting point in Hermarchus' testimony is the division between different ways of understanding legal rules, associated with different types of rationality within the same population. We therefore propose to examine this thesis of an intellectual inequality of citizens, trying in particular to identify some of its axiological consequences within Epicurean political theory, and suggesting clues for a re-evaluation of the role of the wise man as educator.
\end{abstract}

Keywords: political theory, epicureanism, rationality, justice, law, punishment, inequality

\section{Resumen}

Este artículo tiene como objetivo afrontar una grave dificultad en el pensamiento político epicúreo a través de la investigación de una probable contradicción entre la ambición soteriológica universal del epicureísmo, que se presenta como una sabiduría dirigida a todos y adaptada a todos, y el retrato de una humanidad con diversas capacidades intelectuales, dentro de la cual algunos individuos no podrían lograr una conciencia política activa y autónoma. A pesar de la escasez de las fuentes textuales, algunos elementos de este pensamiento político se conocen gracias a fragmentos del Contra Empédocles de Hermarco, que desarrollan algunas de las hipótesis lapidarias esbozadas por Epicuro al final de las Kuriai Doxai. Un punto particular-mente interesante en el testimonio de Hermarco es la división entre diferentes formas de entender las reglas legales, asociadas con diferentes tipos de racionalidad dentro de una misma población. Por lo tanto, nos proponemos examinar esta tesis de una desigualdad intelectual de los ciuda-danos, tratando en particular de identificar algunas de sus consecuencias axiológicas dentro de la teoría política epicúrea y sugiriendo una reevaluación del papel del sabio como educador.

Palabras clave: teoría política, epicureísmo, racionalidad, justicia, ley, castigo, desigualdad

ПНГН/FONS 5(2020), 39-59

ISSN 2445-2297 www.uc3m.es/pege
J. Giovacchini, La confusión du juste DOI https://doi.org/10.20318/fons.2020.5052 


\section{Introduction $^{1}$}

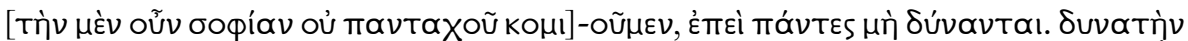

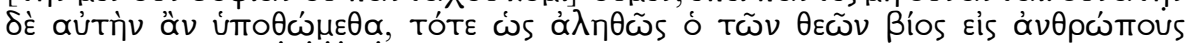

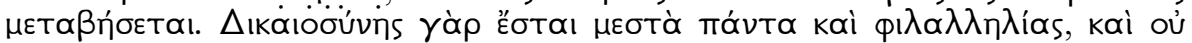

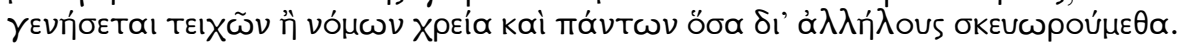

«... puisque tous n'en sont pas capables. Mais supposons qu'elle soit possible: alors, véritablement, la vie des dieux passera chez les hommes. Tout sera, en effet, rempli de justice et d'affection mutuelle, et il n'y aura plus besoin de fortifications ni de lois, ni de tout ce que nous machinons pour nous protéger les uns des autres»².

Telle est la description, proposée par Diogène d'Enoanda dans son inscription, d'une utopie politique rendue possible par la participation de tous à la sagesse d'Épicure. Cette vie de plaisir et d'harmonie, cet âge d'or se caractériserait par une paix universelle rendant caduques les institutions politiques, décrites ici comme uniquement dirigées vers le maintien de la cohésion sociale ${ }^{3}$. Mais d'emblée, Diogène souligne que cet état de paix ne peut sortir de la virtualité; en effet, «tous n’en sont pas capables»».

D’où vient cette incapacité? Si l'on en croit ce fragment, il semblerait que les épicuriens aient adopté, vis-à-vis de la compétence politique, une perspective extrêmement pessimiste; il n'y a aucune expertise politique spontanée et l'esprit humain est plus ou moins apte à saisir les éléments fondamentaux de la justice, sans que cette inaptitude soit réformable. Ce qui n'est pas sans surprendre; cette hypothèse d'une insuffisance gnoséologique de certains hommes, si elle semble en adéquation avec des théories politiques aristocratiques comme celle de Platon, paraît peu cohérente avec le «popularisme» attribué aux sectateurs du Jardin ${ }^{4}$. Le fait est que Platon, dans les Lois, expulse de la Cité les «incurables» et les considère comme exclus de la politique sans espoir de retour'; une telle expulsion s'accorde très mal avec l'intention sotériologique de l'épicurisme et la volonté affichée dans de multiples textes de s'adresser à toute l'humanité pour lui apporter paix et bonheur. Le même Diogène d'Enoanda dans les premiers fragments de son inscription n'écrit-il pas que son discours doit s'adresser «au plus grand nombre», y compris à ceux qui ne sont pas «de bonne composition»?

\footnotetext{
${ }^{1} \mathrm{Je}$ remercie chaleureusement les éditeurs et relecteurs qui m’ont aidé à améliorer cet article; les erreurs et imprécisions qui demeurent doivent m'être attribuées.

2 Diogène D’FEnoanda, fr. 56, éd. SMith (1993), trad. Morel dans Delattre et al. (2010), 1055; Morel relève que selon Smith le début manquant du fragment mentionne la sagesse, cf. Morel dans DELATTRE et al. (2010), 1413 n. 114.

${ }^{3}$ Sur la dimension hobbesienne des intuitions politiques épicuriennes, et la nécessité de relativiser cette catégorisation, voir ROBITZSCH (2017).

${ }^{4}$ Voir par ex Giovacchini et al. (2011).

${ }^{5}$ Cf. Helmer (2017), 140-141, qui explique notamment comment ce filtrage est une dimension essentielle de la théorie politique platonicienne, à l'inverse des thèses démocratiques soutenues par Protagoras.
} 


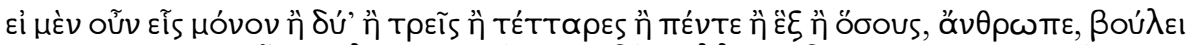

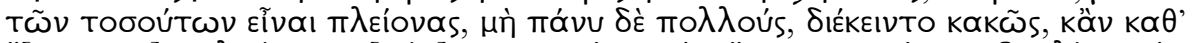

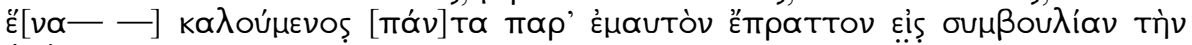
àp̣íotro.

«Si donc il y en avait un seulement ou deux, ou trois, ou quatre, ou cinq, ou six, ou autant que tu veux en plus, cher homme - sans toutefois que leur nombre soit excessif, qui soient dans de mauvaises dispositions, même s'il fallait alors un par un les convoquer [tous], je ferais ce qui est en mon pouvoir pour leur donner le meilleur conseil» ${ }^{6}$.

Il convient donc d'analyser, pour résoudre cette apparente tension, d'une part l'ampleur et la nature de cette insuffisance politique qui oblige l'humanité à recourir aux artefacts que sont les «lois et les fortifications» pour sa survie; d'autre part les solutions éventuelles proposées par l'épicurisme pour pallier cette insuffisance. Ces solutions, on le verra, impliquent une réévaluation du rôle du sage, appelé à remplir une fonction non pas tant politique que pédagogique, donnant une saveur nouvelle à l’impératif de la «vie cachée» et à l'apparent mépris épicurien pour les fonctions officielles au sein de la Cité.

L'hypothèse que les hommes ne sont pas égaux devant l'appréhension des notions nécessaires à la vie politique est d'emblée lisible dans la description même des mécanismes gnoséologiques à l'origine de ces notions, telle qu'on la trouve dans deux textes essentiels: d'une part, l'ensemble formé par les Maximes Capitales d'Épicure 31 à 40, d'autre part la généalogie du droit attribuée à Hermarque et transmise par le $D e$ abstinentia de Porphyre ${ }^{7}$. Ces deux textes définissent le mécanisme mental en question sous le terme technique de prolepsis ou prénotion.

Épicure signale très explicitement dans les Maximes Capitales que le juste, défini comme «sumbolon tou sumpherontos» ${ }^{8}$, est l'objet d'une prénotion et que cette prénotion évolue dans le temps - ce qui distingue d'emblée la prénotion de l'intuition stable et définitive d'une notion objective.

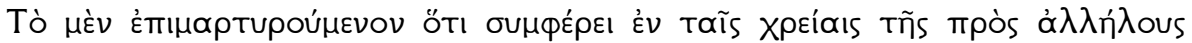

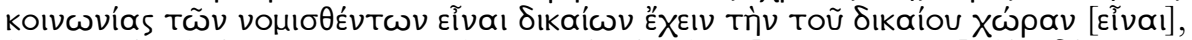

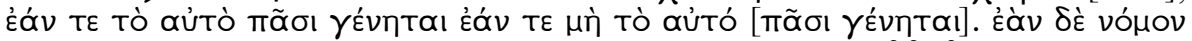

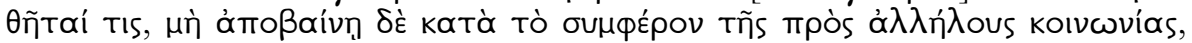

\footnotetext{
${ }^{6}$ Fragment 3, éd. Smith (1993), trad. Morel dans Delattre et al. (2010), 1029.

${ }^{7}$ La critique a pris l'habitude d'identifier dans cette longue citation de Porphyre, qui occupe plusieurs pages (I 7-12), un reste du Contre Empédocle d'Hermarque. Sur cette question d'attribution on pourra se reporter à OBbink (1988) et la préface de LONGO AURICCHIO (1988). Porphyre lui-même, au début et à la fin de la citation, se contente de mentionner «les épicuriens»; mais plus loin dans le traité, en I 26, 4, Hermarque est cité nommément.

${ }^{8} M C$ XXXI.
} 


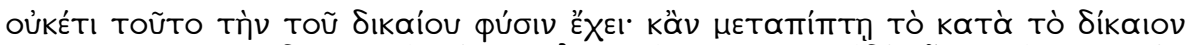

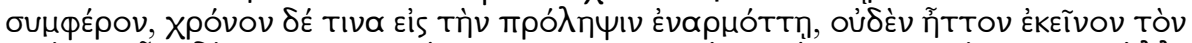

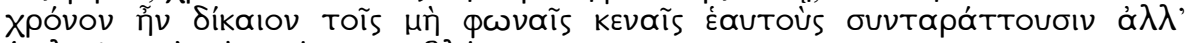

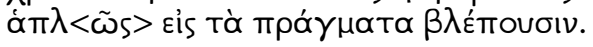

«XXXVII Ce qui est confirmé comme utile dans les usages de la communauté des hommes entre eux, parmi les actes que les lois disent justes, occupe la place du juste, qu'il soit ou non le même pour tous. Et si quelqu'un édicte une loi qui n'aboutit pas à quoi que ce soit d'utile dans la communauté mutuelle, <cette loi> n'aura plus la nature du juste. Et si l'utile devient opposé au juste, et met un moment à s'adapter à la prénotion, il n'est pas moins juste pendant ce moment pour ceux qui ne se laissent pas troubler par des sons vides, mais qui attachent leur regard uniquement aux réalités».

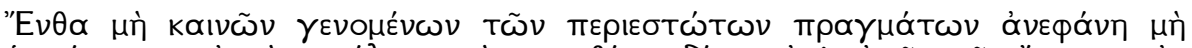

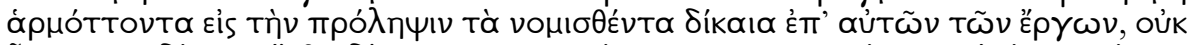

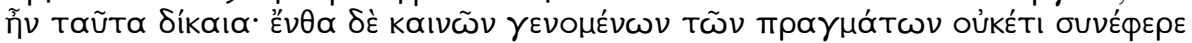

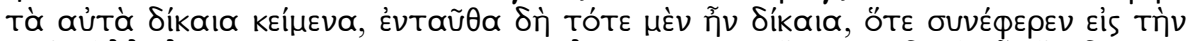

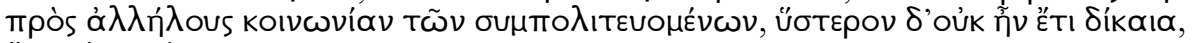

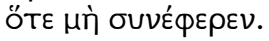

XXXVIII Dans le cas où il ne s'est rien produit de nouveau dans l'état général des affaires, si ce que la loi dit juste ne s'adapte visiblement pas avec la prénotion < du juste> à propos des mêmes actions, alors cela n'est pas juste. Et dans le cas où il s'est produit du nouveau, si ce qui a été déterminé juste n'a plus d'utilité, alors cela était bien juste tant que cela était utile à la communauté mutuelle des concitoyens; et cela n'a plus été juste lorsque cela n'a plus été utile ${ }^{9} \gg$.

Dans ces deux brefs paragraphes, Épicure arrive en quelque sorte au sommet de ses hypothèses sur la nature et l'histoire du droit, en proposant l'analyse, dans une langue très difficile, d'un cas-limite qui est justement une source de haute confusion: le cas où la prénotion du juste et le sentiment de l'utile entrent en discordance. Ce caslimite va notamment être utilisé pour dégager la nature exacte de la distinction entre le juste et l'utile, et pour hiérarchiser conscience du juste et sentiment de l'utile au sein du processus socio-politique général qu’il décrit.

Pour comprendre les implications de ces deux Maximes, il convient d'abord de faire un point sur le terme même de prénotion. Il s'agit d'une réalité mentale, décrite avec soin par Diogène Laërce (X 33) dans son résumé du Canon d'Épicure comme le plus complexe des critères de vérité. La prénotion fonctionne à la manière d'une image mentale, qui a la particularité de renvoyer à un contenu notionnel toujours vrai - et de ce fait, susceptible d'un usage critérial exactement au même titre que les sensations, premiers critères de vérité pour Épicure. Ce qui n'est pas sans soulever une difficulté majeure: dans la mesure où la prénotion est bien le produit d'une construction, de ce fait discursive et située dans le temps, comment peut-elle jouer un rôle similaire à celui de la sensation qui fonde sa valeur critériale sur son alogicité et son immédiateté, c'est à dire sur le fait d'être donnée et non forgée? Le mécanisme de formation des

${ }^{9}$ Éd. Marcovich (1999), nous traduisons. 
prénotions, tel que décrit par Diogène Laërce, est de ce fait celui d'une synthèse empirique, qui s'appuie principalement sur la sollicitation de souvenirs d'impressions passées, sans faire appel au logismos discursif, ou seulement de façon minimale ${ }^{10}$.

Une différence essentielle entre la prénotion et tout autre type de concept réside donc dans sa temporalité: la prénotion est dans le temps, car elle est à la fois une réalité mentale mais aussi le processus de production même de cette réalité, parce qu'elle est de nature synthétique ${ }^{11}$. En, d'autres termes, elle est le produit d'un travail de l'esprit appuyé sur la mémoire, toujours susceptible d'enrichissements et de modifications.

Cet effort de l'esprit est ce qui crée une tension dans les Maximes 37 et 38, et un décalage entre différents niveaux d'appréhension du juste, au sens où le juste ne peut pas être l'objet d'une intuition spontanée accomplie une fois pour toutes. Ces deux maximes décrivent de ce fait une réelle situation de confusion, susceptible de troubler fortement les esprits et de dissoudre la compréhension du juste; elles décrivent le moment où l'équivalence du juste et de l'utile se modifie - où ce qui était utile ne l'est plus, et où donc la nature même du juste intrinsèquement similaire à l'utile se transforme. Ce qui nous indique donc que le travail de la prénotion est bien un travail d'association du juste et de l'utile dépendant de circonstances extérieures à l'intuition elle-même, et que cette association est susceptible de subir des modifications au gré des changements de circonstance ${ }^{12}$. La confusion émergerait alors dans l'esprit de ceux qui seraient amenés à confondre le juste en tant que prénotion - c'est à dire synthétiquement lié à l'utile - avec les manifestations légales ponctuelles du juste. Ces individus, inconscients du travail mental de la prénotion sous-jacent à l'énoncé des lois elles-mêmes, seraient alors perturbés par la bascule sensible entre ce qui serait encore dicté par les lois, en décalage avec la prénotion, et ce qui apparaîtrait comme davantage conforme à l'utile et pourtant hors-la-loi.

Cette situation de confusion, en plus de faire ressortir la temporalité réelle du travail de la prénotion, crée également une hiérarchie entre le juste et l'utile hiérarchie qui était déjà contenue en germe en $M C 31$, dans l'image du sumbolon.

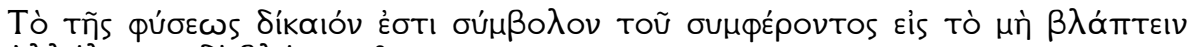

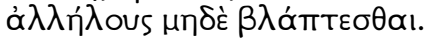

«XXXI Le juste naturel, c'est ce qui permet d'identifier (sumbolon) ce qui est utile pour ne pas se nuire mutuellement ni subir de nuisances» ${ }^{13}$.

10 Sur la prénotion épicurienne en général, voir GLIDDEN (1985), Morel (2008), EssLer (2011), 148-187, GIOVACCHINI (2012), 29-37, TSOUNA (2016). Sur la prénotion juridique voir plus précisément le premier chapitre de GOLDSCHMIDT (1977), et MOREL (2000), 406-407.

11 GoldSCHMIDT (1977), 29 décrit la prolepse comme une «abstraction», un «mouvement de l'esprit».

12 Morel (2000), 409: «Quant à la prolepse du juste, elle ne doit pas être comprise comme une définition immuable du juste, mais comme un simple cadre de variation. À la fois générale et relative, latente et actuelle, elle est l'expression même de la dimension elle-même «actuelle», au double sens du terme, de la réalité politique».

${ }^{13}$ Éd. Marcovich (1999), nous traduisons. 
Beaucoup a été écrit sur la première partie de cette maxime, notamment sur l'énigmatique expression «to tès phuseos dikaion» - et plus généralement, sur la question de savoir si, à partir de cet énoncé, on peut ranger Épicure plutôt parmi les naturalistes ou les conventionnalistes. Ce débat nous entraînerait trop loin ${ }^{14}$; nous nous intéresserons plutôt ici à la deuxième partie de la phrase, et à l'expression de «sumbolon de l'utile» dont nous proposons une traduction qui explicite le sens que nous donnons au sumbolon. L'image mobilisée ${ }^{15}$, telle que nous la comprenons, est complexe; elle implique d'une part qu'il est impossible que le juste et l'utile soient indépendants par nature (l'un ne peut pas ne pas être lié à l'autre d'une façon ou d'une autre, mais c'est leur relation qui leur donne sens), d'autre part qu'ils sont mis à distance l'un de l'autre - on ne les a pas en même temps sous les yeux, sauf par un rapprochement volontaire. Ils sont en quelque sorte essentiellement liés mais pragmatiquement séparés ${ }^{16}$. Il nous semble qu'on peut ici tout à fait rapprocher cet usage du sumbolon de l'usage aristotélicien du début du De Interpretatione: ce qui caractérise le sumbolon c'est à la fois sa fonction de signe et sa nature conventionnelle. Aristote fait du signe écrit le sumbolon toujours provisoire du son signifiant (la parole), car l'écriture varie dans les lieux et les temps; c'est la relation occasionnelle, donc arbitraire, entre sumbolon et phonè qui fonde la signification et la soutient, le sumbolon étant nécessairement second par rapport à la phonè ${ }^{17}$.

On va retrouver cette dissymétrie du symbolisé et du symbole dans le cas de la relation du juste et de l'utile; car, s'il y a prénotion, donc discursivité et évolution du juste, rien de tel pour ce qui est de l'utile. L'utile n'est pas l'objet d'une prénotion, mais d'une intuition spontanée, universelle et définitive; en ce sens l'utile est premier, et le juste n'en est que le sumbolon ou signe de reconnaissance. Encore faut-il s'entendre sur ce dont il est ici question quand on parle d'utile.

Cette nature intuitive et nécessaire de l'utile est en réalité un prérequis éthique important dans la doctrine épicurienne; on peut la retrouver en filigrane dans l'argument dit «des berceaux», transmis par Cicéron dans la bouche de l'épicurien Torquatus, et qu'on peut résumer ainsi: tout être vivant sait spontanément ce qui est bon pour lui, sans qu'il y ait besoin pour cela d'argument ou de démonstration - car «cela se sent»:

${ }^{14}$ Morel (2000), 396, fournit une synthèse de ces débats.

${ }^{15}$ Le sumbolon désigne depuis l'époque classique la tessère d'hospitalité, brisée en deux, partagée et pouvant servir de signe de reconnaissance entre deux individus; Louis Gernet a étudié les évolutions de sens de ce terme qui a peu à peu fini par devenir un équivalent matériel du contrat coutumier ou de l'accord conventionnel. Voir par exemple GERNET (1928), 349.

${ }^{16}$ En ce sens, l'image des deux faces du même objet, reprise par Morel (2000), 402, nous semble trompeuse. Historiquement, le sumbolon est un objet brisé dont on peut ensuite réunir les deux parties. La mise côte à côte demeure possible même si elle n'est jamais effectivement réalisée - contrairement à la pièce de monnaie, dont les deux faces ne peuvent jamais être vues ensemble.

${ }^{17}$ La comparaison a ses limites car Aristote utilise dans ce contexte sumbolon pour désigner des signes explicitement et uniquement de nature linguistique, (ce que rappellent par exemple POLANSKYKUCZEWSKi 1990), ce qui n'est pas le cas d'Epicure. Mais la relation dans les deux cas nous semble de même nature et c'est sur cette relation que porte le rapprochement que nous effectuons. 
Omne animal, simul atque natum sit, voluptatem appetere ea que gaudere ut summo bono, dolorem aspernari ut summum malum et, quantum possit, a se repellere, id que facere nondum depravatum ipsa natura incorrupte atque integre iudicante. itaque negat opus esse ratione neque disputatione, quam ob rem voluptas expetenda, fugiendus dolor sit. sentiri haec putat, ut calere ignem, nivem esse albam, dulce mel. quorum nihil oportere exquisitis rationibus confirmare, tantum satis esse admonere.

«Tout être animé, dès sa naissance, recherche le plaisir et s’en réjouit comme étant le bien suprême, mais il abhorre la douleur comme étant le mal suprême et la repousse autant qu'il peut. Il se comporte ainsi quand il n'est pas encore perverti, la nature ellemême prononçant un jugement intègre et incorruptible. [Épicure] soutient donc qu'il n'est pas nécessaire de raisonner et de discuter pour savoir pourquoi le plaisir doit être recherché et la douleur évitée. Il pense que cela se sent, comme on sent que le feu est chaud, la neige blanche, le miel doux: il n'y a pas besoin de raisonnements sophistiqués pour le confirmer, il suffit de le faire remarquer» ${ }^{18}$.

La conscience du telos, c'est à dire de ce qui doit fondamentalement être recherché, est sans enseignement et sans effort: elle est sentiment de ce qui est bon pour soi, pathos fondamental, résumé sous le terme de plaisir, qui renvoie dans l'économie générale de l'éthique épicurienne aussi bien à ce qui est plaisant que, et surtout, à ce qui va préserver l'intégrité morale et physique de l'individu - à ce qui va le maintenir en cohésion avec lui-même. La nature est, pour tout être sensible, organisée de façon bipolaire, entre éléments attirants (objets de choix) et éléments répugnants (objets de rejet), les choix et les rejets étant subordonnés à l'impératif absolu de se maintenir en bon état - et le sentiment de plaisir étant considéré comme le signe le plus sûr du bon état général du vivant. En ce sens, il n'y a pas de prénotion de l'utile comme il n'y a pas de prénotion du plaisant ou de l'agréable - car il n'y a aucune synthèse empirique à l'œuvre dans l'identification du plaisant ou de l'agréable, mais la sensation immédiate d'un soulagement ou d'une plénitude satisfaisante qui n'est pas susceptible d'évolution ou d'amélioration. Le plaisir est toujours reconnu, ce qui signifie qu'il est d'emblée archaïquement et fondamentalement connu.

Mais il est nécessaire de nuancer ce tableau éthique; en effet, l'argument des berceaux nous présente une humanité dans son état premier, l'état d'enfance, arationnel, non encore infecté par le jugement ou les artifices culturels. Hors de cet état d'enfance, la perception du telos est brouillée et les hommes confondent très régulièrement le plaisant et le déplaisant, faute de pouvoir se fier à leurs impressions. C'est ce qui justifie pour Épicure la parole philosophique elle-même: il s'agit de redonner à l'humanité la capacité à se repérer dans la nature selon la bipolarité du plaisant et du déplaisant, afin qu'elle puisse effectuer de façon sensée les choix et les rejets qui garantiront sa sécurité.

C'est aussi le cas pour le droit: alors que le sentiment de l'utile est un pathos, donc irrationnel et universel en théorie, il se trouve obscurci en pratique par les jugements

${ }^{18}$ Cicéron, De Finibus I, IX 30, éd. Schiche (1915), trad. Kany-Turpin (2016). 
et opinions vides et doit donc être signalé, marqué par un sumbolon qui sera la prénotion du juste ${ }^{19}$. Mais on voit en suivant les Maximes que, dans le cas du droit, on a en réalité affaire à deux niveaux «symboliques»: car si la prénotion du droit signale l'utile, elle-même est formalisée dans les communautés humaines par la loi, qui fixe en quelque sorte pour une société donnée la relation du juste et de l'utile nouée par la prénotion. Il y a donc plusieurs niveaux de complexité, et par là, plusieurs occasions de confusion.

Ces niveaux de complexité, ramassés chez Épicure dans des Maximes d'une densité qui en augmente l'obscurité, sont heureusement pour nous développés et en quelque sorte dépliés dans la généalogie du droit attribuée à Hermarque par Porphyre au premier livre du De abstinentia. L'approche généalogique lui permet d'expliciter ces différents moments de confusion, et de les attribuer à différentes catégories d'individus chez qui la confusion ne s'exprimera pas de la même façon et n'appellera pas les mêmes solutions ${ }^{20}$.

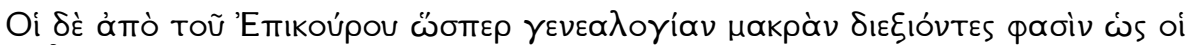

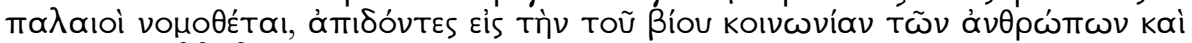

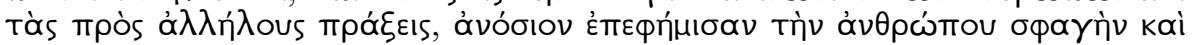

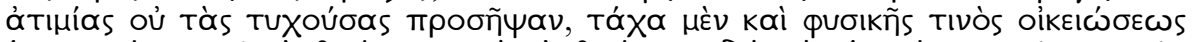

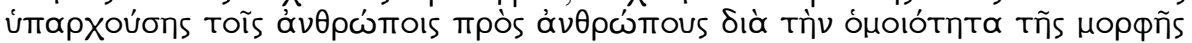

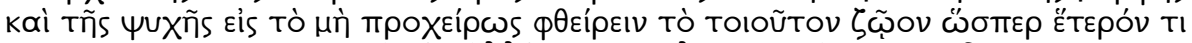

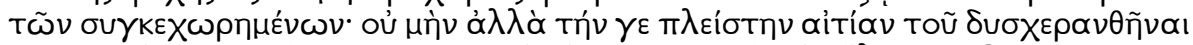

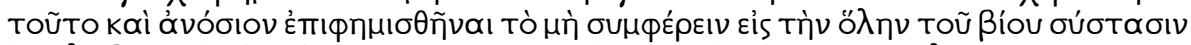

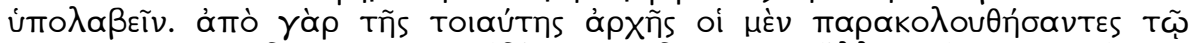

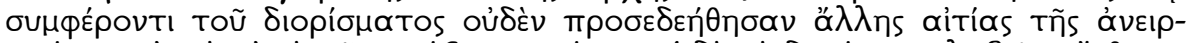

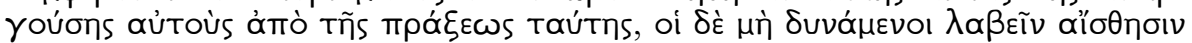

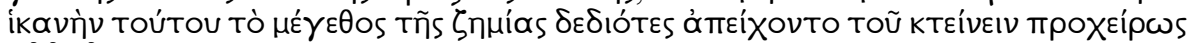

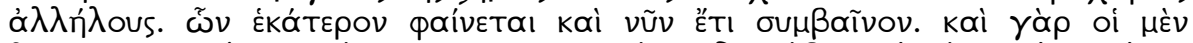

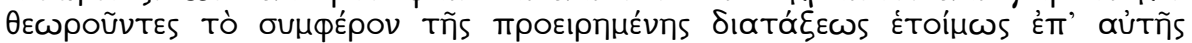

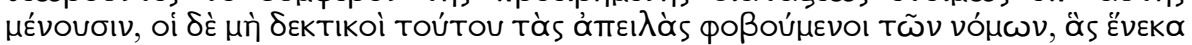

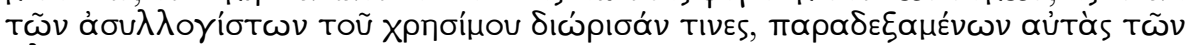
$\pi \lambda \varepsilon \varepsilon^{\prime} v \omega \nu$.

\footnotetext{
19 Nous ne suivons pas Morel (2000), 397 qui fait de «l'utile commun» une réalité «variable dans ses manifestations et naturel[le] dans son principe»; en effet, si notre compréhension de la relation du juste à l'utile comme à son sumbolon est exacte, c'est le juste qui peut être susceptible de variation, jamais l'utile; car c'est le signe qui peut conventionnellement changer, mais pas le signifié. L'utile en tant que pathos demeure le même, de même que le plaisir qui, en tant que souverain bien, peut varier en intensité mais pas en nature - mais le pathos lui-même peut être le produit de démarches et de choix variés selon le lieu et le moment. C'est probablement un vrai point de fragilité dans l'édifice conceptuel épicurien, car il est bien difficile de soutenir ainsi que l'utile en tant que pathos est un invariant humain; mais il faut bien admettre que c'est ce que semble nous dire le texte des Maximes, et que nous ne pouvons ici sauver les épicuriens d'eux-mêmes.

${ }^{20}$ Pour une analyse globale de cette généalogie, voir en priorité VANDER WAERDT (1988).
} 
«Ceux qui suivent Épicure disent, en développant ce qui ressemble à une vaste généalogie, que les anciens législateurs, ayant observé de près la communauté de vie des hommes et leurs conduites les uns envers les autres, ont dénoncé comme impie le meurtre d'un homme et lui ont attaché des châtiments bien spécifiques ${ }^{21}$; tout d'abord ils identifièrent rapidement une certaine appropriation des hommes avec les hommes, du fait de leur ressemblance de forme et d'âme, et en conclurent qu'il ne fallait pas immoler témérairement ce genre de vivant, en le confondant avec les vivants pour lesquels cela est admis; néanmoins <ils disent que> selon ces législateurs la raison principale du fait que cela soit considéré comme insupportable et dénoncé comme impie est que cela était désavantageux pour l'organisation générale de la vie. À partir de ce principe, ceux qui avaient compris l'avantage de cette décision n'eurent besoin d'aucune autre raison pour s'écarter de cette conduite, mais ceux qui ne pouvaient pas en avoir une sensation suffisante, craignant l'importance du châtiment, s'abstinrent de se tuer témérairement les uns les autres. Aujourd'hui encore, on observe chacune des deux attitudes. Et en effet ceux qui ont l'intuition du caractère avantageux de la convention décrite plus haut s'en tiennent à elle avec zèle, mais ceux qui ne sont pas capables de l'appréhender craignent les menaces des lois, qui ont été fixées à cause de ces individus dépourvus de la compréhension de l'utile, celle-ci ayant été acceptée par la plupart $<$ des hommes $>»^{22}$.

Le texte d'Hermarque est rapporté par Porphyre dans une intention bien particulière, puisqu'il s'agit de rapporter ce qui, dans les conceptions épicuriennes, leur autorise la consommation de viande, donc le meurtre animal, mais leur interdit le meurtre humain. L'argumentation ici restituée par Porphyre porte donc sur un élément juridique particulier et d'une importance maximale puisqu'il est présenté comme un prérequis social: aucune communauté humaine ne peut survivre sans s'être prémunie contre le meurtre ${ }^{23}$.

L’argument épicurien est développé sur plusieurs pages. Les deux paragraphes dont nous proposons ci-dessus une traduction en présentent une première version synthétique qui nous suffira dans le cadre de cet article pour analyser le point précis qui nous occupe; la suite du texte reprend et développe ces éléments en insistant d'une part sur la justification de la violence du châtiment pour entraîner l'adhésion de tous, y compris de ceux qui ne parviennent pas à comprendre le bien fondé de la loi, d'autre part sur l'autorisation du meurtre animal qui, lui, ne nuit pas à la communauté mais au contraire lui est utile.

${ }^{21}$ Le meurtre est présenté par Hermarque comme un cas-limite, et son interdiction, une condition de possibilité de la vie politique. Cela lui donne un statut juridique particulier. Nous choisissons de traduire ici atimias ou tas tuchousas par «châtiments bien spécifiques» au lieu de la proposition de Bouffartigue, «indignités exceptionnelles»: il s'agit en effet pour Hermarque non pas de mettre en valeur l'ampleur des châtiments ou leur rareté, mais leur caractère inouï. C'est à partir de la question du meurtre en effet que les premiers législateurs font vraiment œuvre principielle, œuvre de fondation. Ils n'améliorent pas le droit existant ni ne l'amplifient, mais ils l'instaurent en faisant basculer l'humanité dans la vie politique.

22 De Abst. I 7, éd. Bouffartigue (1977), nous traduisons.

23 BESNIER (2001), 135-136 insiste ainsi à juste titre sur le caractère premier et fondateur de cette législation, qui crée pour le nomothète une double difficulté: inventer un cadre juridique inédit et l'imposer à ceux qui n'en comprennent pas la nécessité. 
D'un point de vue lexical, on relève d'emblée que le terme de sumbolon n'apparaît nulle part; mais le développement fait en revanche plus loin intervenir un terme nouveau, celui de pharmakon, dans un contexte qui semble le rapprocher fonctionnellement du sumbolon, en tant qu'intermédiaire entre deux niveaux de compréhension: «car il n'y avait pas d'autre remède (pharmakon) contre l'ignorance de l'utile que la peur du châtiment prescrit par la loi» ${ }^{24}$.

Les individus faibles intellectuellement (ce que connote le terme choisi pour désigner leur ignorance, amathia) ont besoin de quelque chose qui puisse jouer le rôle de sumbolon de l'utile; car ils sont dans un tel état d'incompréhension qu'il leur est impossible d'accéder à la prénotion du juste, et donc, à la connaissance de l'utile qui est sa conséquence. Se substitue donc au sumbolon le pharmakon, en l'occurrence ici la peur du châtiment; mais la relation ici, contrairement à la précédente, n'a plus rien de symbolique. Là où le juste signalait l'utile et en permettait la compréhension, la peur vient remplacer la compréhension, devenue impossible, et substitue donc un pathos réceptif à une anticipation active comme l'est la prolepsis. Ce qui est une autre façon de dire que l'individu faible d'esprit, malade, ne peut avoir qu'un rapport réactif et passif à la loi.

Toujours sur le plan lexical, la notion de justice quant à elle n'intervient qu'à la toute fin du développement:

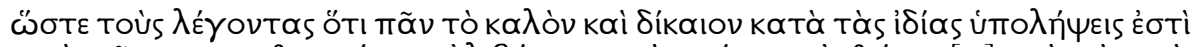

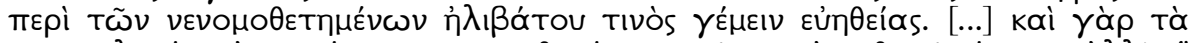

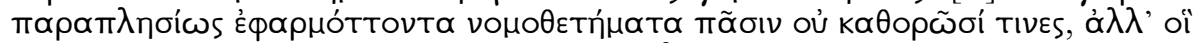

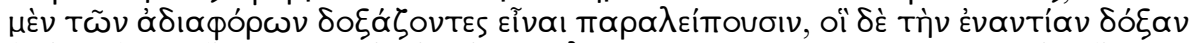

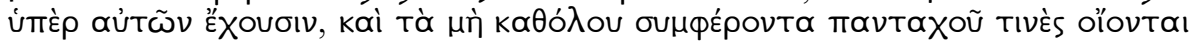

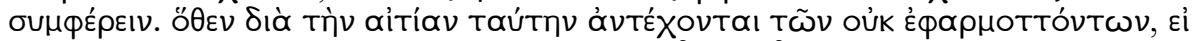

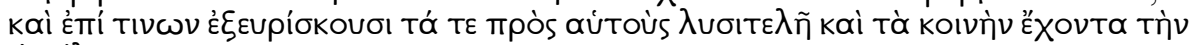

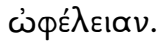

«De sorte qu'ils sont boursouflés de bêtise, ceux qui disent, à propos des législations, que le beau ou le juste (dikaion) sont toujours dictés par des intuitions (hupolepseis) singulières. [...] Car certains ne voient pas les législations qui sont à peu près pareillement adaptées à tous, mais soit les négligent en les tenant pour indifférentes, soient les tiennent au contraire pour supérieures et, alors qu'elles ne sont pas universellement utiles, les pensent utiles partout. Pour cette raison ils s'attachent à des législations qui ne leur sont pas adaptés, même si leur intelligence comprend ce qui est dans leur intérêt individuel ou ce qui est utile à la collectivité» 25 .

Il n'est pas dans ce passage question de la justice en elle-même, en tant que catégorie indépendante; mais le texte souligne, en maintenant une subtilité remarquable dans son analyse, d'une part la relativité irréductible du juste en tant qu'il demeure subordonné à une utilité variable en temps et en lieu, d'autre part l'invariabilité de certaines prescriptions que l'on va retrouver à l'identique ou

${ }^{24}$ I 8,3 .

${ }^{25}$ I 12, 2-3, éd. Bouffartigue (1977), nous traduisons. 
quasiment (le texte insistant sur cette nuance) d'un groupe à l'autre, le travail de l'intelligence consistant d'abord dans la capacité à adapter avec souplesse et rigueur la législation à la situation.

Sont conservés par rapport à l'exposé juridique des Maximes les termes liés à la légalité (nomos et sunthèkè) et à l'utilité (sumpheron, chrèsimon); le terme même de prolepsis est absent, mais figure à plusieurs reprises dans le texte celui d'epilogismos, procédure rationnelle empirique ${ }^{26}$ qui joue ici un rôle noétique tout à fait important et très proche de celui de la prénotion, en ce qu'elle décrit exactement le mécanisme mental par lequel les individus comprennent le bien-fondé de la loi en mettant en relation l'interdit qu'elle énonce avec le rôle qu'elle remplit vis à vis de l'intérêt collectif - rôle proprement symbolique donc de mise en relation du signifiant qu'est le nomos avec le signifié qu'est le sumpheron. Au contraire, l'hupolepsis apparaît textuellement pour désigner la supposition personnelle, non appuyée sur la synthèse de l'epilogismos - une croyance ou opinion idiosyncrasique qui ne peut pas jouer de rôle symbolique car elle demeure strictement individuelle.

Le texte fait intervenir comme protagonistes de cette généalogie du droit les «anciens législateurs», nomothètes à l'origine de l'interdit fondamental du meurtre. Contrairement à la mise en scène qu'on peut lire par exemple dans le mythe de Protagoras tel que rapporté par Platon dans le dialogue éponyme, le droit est une technique, non pas providentielle, offerte ou dictée par la divinité, mais produite par l'intelligence humaine contrainte par une situation naturellement inconfortable. Selon un schéma anthropologique connu et attesté dans de multiples sources, la nature épicurienne se corrige en quelque sorte elle-même; elle n'est pas spontanément appropriée à l'homme, mais s'instruit elle-même ${ }^{27}$ et s'amende par le biais des techniques qui ne sont pas apportées de l'extérieur à l'humanité mais qui procèdent d'un aménagement par l'homme de son environnement, aménagement d'abord grossier puis de plus en plus raffiné28.

Néanmoins cet aménagement n'est pas le fait de tous les hommes, mais de certains, plus doués que les autres, qui font preuve d'un génie particulier. Or, la première technologie juridique, l'interdit du meurtre, est présentée par Hermarque comme axiologiquement première: elle est la condition initiale sans laquelle le groupe humain ne peut subsister. On se retrouve donc dans une situation anthropologiquement contradictoire: si tout homme, selon les principes de l'éthique épicurienne, sait par nature ce qui est bon pour lui et sa survie, au niveau collectif cette intelligence semble défaillante, et seuls quelques individus particulièrement doués sont capables de discerner ce qui va aller dans le sens de la survie du groupe. Dans la mesure où ces premiers législateurs interviennent au sein des toutes premières sociétés (puisqu'il

\footnotetext{
${ }^{26}$ Sur cette notion méthodologique complexe nous renvoyons à SCHOFIELD (1996), et GIOVACCHINI (2012), 139-161.

${ }^{27}$ Cf. Epicur. Ep. 2, 75, où ce mécanisme de correction immanente est bien décrit à propos de l'origine du langage.

${ }^{28}$ Cf. MOREL (2016).
} 
s'agit bien d'une généalogie, et qu'on va donc remonter à la source même du droit), l'argument de la corruption du jugement est faible. S'il est possible de mettre les erreurs individuelles de jugement sur le telos sur le compte des préjugés dictés par la culture et la dégénérescence humaine, il est difficile de considérer que les hommes avant toute vie sociale sont victimes de cet obscurcissement intellectuel. On doit donc admettre que l'intelligence politique n'est pas de même nature que l'intelligence éthique; elle ne procède pas d'une intuition humaine naturelle et spontanée, mais elle relève d'une capacité intellectuelle susceptible de variations d'un individu à l'autre ${ }^{29}$. Cette différence anthropologique est liée à la multiplication des niveaux de complexité déjà relevée dans les Maximes, et qui apparaît dans ce nouveau texte avec plus de précision. En effet, la généalogie d'Hermarque souligne bien l'absence de rapport direct entre le légal et l'utile, et la nécessité de les relier conventionnellement, la justice étant finalement le nom donné à cette convention. Là où n'importe quel individu, même peu doué, sait reconnaître son intérêt personnel, la reconnaissance de l'intérêt collectif va supposer une compréhension de cette convention, qui va mettre en jeu des qualités d'abstraction et d'anticipation plus subtiles. Si donc la justice, c'est à dire la relation conventionnelle qui unit le légal et l'utile, est facilement appréhendable pour le sage, elle ne l'est pas pour les autres hommes. Les lois interviennent pour aider les hommes peu doués à se soumettre à cette convention.

Ce qui fait qu'on a en réalité affaire, non pas à deux mais à trois niveaux de compréhension à l'œuvre au sein du groupe humain:

- maximal: celui qui a accès à la véritable prénotion du juste, qui est donc capable à la fois d'associer le juste et l'utile ici et maintenant et de le faire avec une souplesse telle qu'elle va anticiper les variations possibles et s'y adapter. Seuls ces individus ont les capacités pour être législateurs.

- intermédiaire: celui qui a compris la relation entre le juste et l'utile mais qui va la maintenir figée, et sacraliser en quelque sorte la loi - au lieu de ne voir dans la loi que la trace d'une relation momentanée entre le juste et l'utile. Celui-ci n'a pas besoin à proprement parler de la peur du châtiment, mais il a perdu la plasticité de la prénotion et peut donc se trouver embarrassé pour identifier les moments où le légal n'est plus le juste.

- bas: celui qui est incapable de saisir la prénotion parce qu'il ne comprend pas le lien du juste et de l'utile et croit que son intérêt est de ne pas suivre le juste; il a donc une mauvaise compréhension de son propre intérêt, due à un manque cognitif qui repose d'abord sur une incapacité à anticiper et à mettre en relation ${ }^{30}$. Il pose un

\footnotetext{
${ }^{29}$ On trouve d'autres occurrences d'une telle partition dans le corpus épicurien; sans les citer toutes, on peut pointer particulièrement Lucr. V 1105; D.L. X 117; Diog. Oin. fr. 39 et 44.

30 BESNIER (2001), 143 semble rabattre l'une sur l'autre les deux catégories des moins avancés et des définitivement incapables: «ce n'est pas qu'une pareille chose soit contraire à leur nature (si elle l'était, les législateurs devraient imposer la décision par la force, ce qu'Hermarque écarte précisément), c'est simplement qu'en eux la nature est déficiente et qu'ils ne saisissent pas tout de suite l'utile, ou ne stabilisent pas cette appréhension». Il y a confusion ici entre force et contrainte; si tous les citoyens finissent par consentir à la loi, ce consentement doit être obtenu par le recours à la violence légitime (le
} 
problème spécifique, en pratique résolu par le châtiment, solution pour que cet individu puisse demeurer dans la société. On va ainsi remplacer la prénotion, donc l'anticipation de ce qui est bon, par un pharmakon, la peur, c'est à dire l'anticipation de ce qui est mauvais; le châtiment est une violence paradoxale indispensable pour ne pas exclure ces personnes du contrat social. La peur de la souffrance leur donne une forme subalterne et atténuée mais efficace de «symbole de l'utile» qui rend possible avec eux un pacte. Or c'est la capacité ou l'incapacité à nouer un pacte qui fonde la possibilité d'une relation politique avec un être. Sans peur du châtiment, pas de pacte, pas de contrat, pas de politique.

Il nous reste alors à élucider ce qui demeure pour le moment comme une aporie: à savoir la difficile conciliation de ce recours au pharmakon de la peur avec la volonté émancipatrice de l'épicurisme. Dans l'édifice général de l'éthique épicurienne, la peur est un pathos négatif dont il convient de se défaire pour atteindre la vie heureuse, caractérisée au premier chef par l'ataraxia ou absence de trouble. Sur le plan sémantique, la doctrine épicurienne associe systématiquement la sagesse à l'élimination des peurs archaïques (peur des dieux, peur de la mort), présentées comme vides de sens et toujours nuisibles ${ }^{31}$.

On peut supposer dans un premier temps qu'il convient de distinguer ces peurs irrationnelles, qui sont des opinions vides, de la peur du châtiment réel infligé par la loi, rationnellement fondée. Si les châtiments du Tartare ne sont pas à craindre, c'est d'abord parce qu'ils n'auront jamais lieu, la mort supposant la fin de la sensibilité; mais la condamnation pour meurtre entraînera des désagréments bien sensibles, et la salvation épicurienne ne s'adresse donc pas à ces peurs-là.

Mais l'argument est faible. On ne peut qu'être touché par la proximité lexicale, troublante, entre la tetrapharmakos ou quadruple remède éthique, et la qualification par Hermarque de la peur du châtiment comme d'un pharmakon. Ce qui indique que non seulement l'épicurisme n'a pas vocation à détruire toutes les peurs, mais qu'il admet le caractère sain et utile de certaines peurs sans lesquelles il n'est pas de vie collective possible. On peut en inférer que la véritable partition ne s'effectue pas entre certains types de peurs, mais certaines conditions d'existence dans lesquelles la peur est plus ou moins nécessaire. Alors que le quadruple remède éthique s'adresse à l'intime de l'homme, et cherche sa sauvegarde individuelle, la peur du châtiment

châtiment) pour ceux qui ne sont pas capables de s'y soumettre autrement. Le fait que ce consentement soit bien volontaire ne doit pas nous faire oublier qu'il est obtenu par un recours à un pathos, la peur. L'adhésion de celui qui rationnellement comprend le bien-fondé général de la loi n'est pas de même nature que celle de l'individu qui obéit car il craint pour sa vie. Dans le premier cas, il y a une saisie de l'utile collectif grâce à la puissance éducative de la loi; dans le second cas, la saisie de l'utile collectif n'a jamais lieu, seul l'utile pour soi est perçu.

${ }^{31}$ Sur cette question on consultera prioritairement WARREN (2009), et MiTSIS (2014), notamment 77 n. 67. 
concerne l'individu au sein du groupe, dans sa vie publique, dans un espace commun (koinos) hostile strictement opposé à la sécurité de l'espace privé (idios). Il faut nécessairement admettre que ce changement de lieu d'exercice, entre l'espace privé de la philosophie et l'espace public de la politique, suffit à transformer radicalement l'homme et sa relation au monde, au point que ce qui est amendable ou éducable dans le premier espace ne l'est plus dans le second. Les hommes sont les mêmes dans les deux cas, mais c'est leur situation qui modifie du tout au tout leur capacité à apprendre et à progresser.

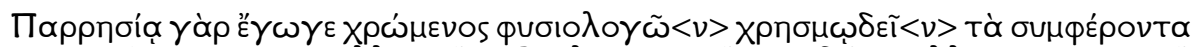

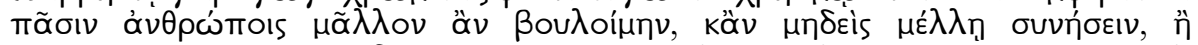

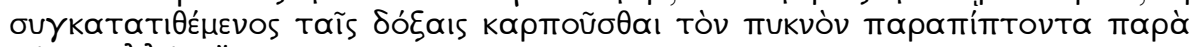

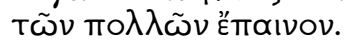

«29. En ce qui me concerne en effet, je préfère user du franc-parler qui caractérise le physiologue et proférer des oracles utiles à tous les hommes, quand bien même personne n'y entendra goutte, plutôt que de me rallier aux préjugés en vogue, et récolter ainsi la louange de la foule» ${ }^{32}$.

La Sentence Vaticane 29 ci-dessus oppose ainsi les «hommes», qui pourraient tous profiter des leçons de l'épicurisme, et ce qui est désigné dans le texte comme «la foule» ou «le grand nombre», l'humanité en tant que collectivité socialisée. La science épicurienne de la nature s'adresse bien à tous en droit, en tant que tous sont des hommes, donc, selon une caractérisation rappelée par Diogène d'Oenoanda, des «êtres de raison $»^{33}$. Mais au sein de la foule l'homme devient irrationnel, superstitieux, et du fait même du rapprochement social est un danger pour son voisin. Épicure distingue deux situations, plutôt que deux catégories d'hommes.

Mais la doctrine épicurienne suppose également que toute l'humanité n'est pas contaminée par la vie sociale et que la confusion mentale qui rend nécessaire l'usage des châtiment n'est pas le lot de tous. Malgré l'inconfort de la collectivité, certains membres de la communauté conservent des capacités intellectuelles qui vont leur permettre d'assurer indirectement la sauvegarde de tous. La différence avec la salvation philosophique va résider dans la nature de l'enseignement proposé. Alors que le sage philosophe souhaite dissiper les craintes irrationnelles en adressant

32 SV 29, éd. MARCOVICH (1999), nous traduisons.

${ }^{33}$ Fr. 21. Le même Diogène souligne d'ailleurs dans un texte à l'argumentation complexe une répartition assez subtile entre ceux qui, ne craignant pas les dieux, agissent correctement parce qu'ils ont «une vision correcte de la nature des désirs, des souffrances et de la mort» et ceux qui le font «à cause des lois et des châtiments qu'elles font peser sur eux» (NF 126, trad. Morel dans DeLATTRE 2010, 10381039). Il s'agit dans ce passage pour Diogène de souligner que l'absence de peur de la mort n'a aucune conséquence politique puisque ceux qui ne craignent pas les dieux ont bien d'autres raisons de ne pas agir injustement, soit de bonnes raisons (la pratique de la physiologie) soit de moins bonnes mais tout aussi efficaces (la peur des châtiments). Ce texte confirme ainsi un traitement clairement différencié entre ces deux peurs respectives, et une corrélation entre la peur du châtiment et la catégorie appelée «gens du vulgaire»; nous remercions grandement un des relecteurs de cet article qui a attiré notre attention sur ce très important fragment. 
directement un contenu doctrinal accessible en théorie à tous, le sage législateur remplit une fonction pédagogique d'une nature tout à fait autre. Cette fonction est historiquement décrite par Lucrèce au chant V du De Rerum Natura, à l'occasion du récit de fondation des toutes premières sociétés. Celles-ci se dotent de rois, choisis parmi les individus les plus forts et les plus physiquement remarquables. Ces rois fondent des entités politiques, organisent la répartition des richesses, puis sont renversés et massacrés ${ }^{34}$.

«Ergo regibus occisis subversa iacebat

pristina maiestas soliorum et sceptra superba, et capitis summi praeclarum insigne cruentum sub pedibus vulgi magnum lugebat honorem; nam cupide conculcatur nimis ante metutum. res itaque ad summam faecem turbas que redibat, imperium sibi cum ac summatum quisque petebat. inde magistratum partim docuere creare

iura que constituere, ut vellent legibus uti.

[...]

inde metus maculat poenarum praemia vitae.

circumretit enim vis atque iniuria quemque

atque unde exortast, ad eum plerumque revertit,

nec facilest placidam ac pacatam degere vitam

qui violat factis communia foedera pacis».

«Donc, une fois les rois tués, gisaient renversés

l'ancienne majesté des trônes et les sceptres superbes,

et l'insigne éclatant de la tête souveraine, ensanglanté,

sous les pieds de la foule pleurait son grand honneur;

car c'est avec avidité qu'on piétine ce qu'on a trop redouté auparavant.

C'est pourquoi les choses en arrivaient à la lie et au désordre extrêmes,

puisque chacun briguait pour soi le pouvoir suprême et la souveraineté.

Alors certains (partim) apprirent aux autres à créer des magistratures

et à établir le droit pour qu'ils acceptent de faire usage de lois ${ }^{35}$.

[...]

Depuis la crainte des châtiments ternit les avantages de la vie.

Car la violence et l'injustice entourent chacun de leurs rets, et d'où qu'elles naissent, elles se retournent le plus souvent contre leur auteur;

et il n'est pas facile de mener une vie calme et apaisée

\footnotetext{
34 Schrijvers (1996) a rapproché l'histoire politique proposée par Lucrèce du metabolè politeion décrit par Polybe au livre VI de son Histoire. Chez Polybe une première royauté dégénère en tyrannie; les rois sont renversés; une aristocratie se met en place, elle-même dégénère à son tour en oligarchie et est renversée; l'avènement de la démocratie prépare l'arrivée de l'ochlocratie (gouvernement de la foule); le désordre général favorise alors la restauration de la royauté, et le cycle recommence ... Un réseau commun d'influence avec Lucrèce semble très probable; cependant il existe une différence massive qui semble donner la clef de la théorie lucrétienne. L'originalité de Lucrèce est en effet d'inverser la dernière étape; c'est l'ochlocratie, dérivée de l'oligarchie qui cède peu à peu sa place à un régime stable de nature républicaine. Ce faisant, il ne semble pas que pour Lucrèce, le cycle puisse recommencer.

35 «Inde magistratum partim docuere creare / iura que constituere, ut vellent legibus uti».
} 
à qui, par ses actes, viole les pactes d'une paix commune» ${ }^{36}$.

Enseignés par ces fameux «partim», les hommes parviennent à instaurer un système de lois, consolidé par l'invention du châtiment, qui doit avoir un effet dissuasif sur ceux qui restent incapables de se soumettre à la loi. Il est bien précisé par Lucrèce que ce système est encore valide au moment où il écrit: «inde metus maculat poenarum praemia vitae». Le système pénal est décrit comme une réutilisation de la violence exercée par les hommes entre eux; cette violence se retrouve détournée justement sur ceux qui cherchaient à l'utiliser contre la cité. La peur est présentée par Lucrèce comme une passion ambivalente: elle bride le bonheur car elle demeure un pathos négatif, mais elle est en même temps la condition de possibilité de la sécurité, dimension essentielle de la vie heureuse ${ }^{37}$; ainsi dans l'espace politique il est impossible de se débarrasser de la peur. Pour transformer l'espace public en espace sûr, il faut en passer par la peur. L'espace public est le lieu d'une promiscuité douloureuse, où se cacher est impossible; mais cette impossibilité de la dissimulation, dangereuse et risquée lorsque les lois n'interviennent pas, devient une bénédiction à partir du moment où la perspective du châtiment lui confère une intention nouvelle. Ne pas pouvoir se cacher se traduit dans l'espace de la cité par une certitude de la punition, et transforme la peur de l'autre, malsaine, en peur de la loi, saine ${ }^{38}$.

La peur du châtiment n'est donc pas irrationnelle, elle est au cœur même de la politique dans la mesure où la vie politique est une mise en danger. L'objet de la loi est de tenir ensemble les hommes en substituant aux liens naturels (l'amitié des premières sociétés, qui disparaît au fur et à mesure que la communauté grandit) ${ }^{39}$ des liens artificiels qui sont ceux de la convention. La peur devient le soutien nécessaire de la loi parce que tout le monde n'est pas ami, au sens où tout le monde n'a pas le sentiment de la communauté, de la ressemblance et de la communauté d'intérêt des uns avec les autres.

Les partim interviennent donc pour éduquer l'humanité à la politique, c'est à dire à la compréhension de l'intérêt collectif. Leur intervention n'a, comme chez Hermarque, rien de providentiel ${ }^{40}$. Mais, contrairement à ce que l'on peut lire chez

${ }^{36}$ DRNV, 1135-1155 éd. Martin (1969), trad. Pigeaud dans Delattre et al. (2010).

37 Cette dimension de sécurité est tellement essentielle à la vie heureuse qu'elle soumet le sage luimême au cadre de la loi. Elle s'impose comme un environnement non négociable hors duquel il est proprement impossible de vivre heureux. De ce fait, l'expérience de Gygès appliquée au sage ne l'amènera pas à enfreindre la loi, malgré son caractère conventionnel - car le sage a justement conscience qu'il convient de s'y soumettre, non pas parce qu'elle serait juste en soi, mais parce que c'est la convention dont elle émane qui assure sa propre sécurité. Cf. entre autres VANDER WAERDT (1987), 421.

${ }^{38}$ Cf. là-dessus notamment AUSTin (2012).

39 MiTsis (2014), 129-130 relève le caractère pacifique des premières associations entre les hommes, à un moment où il n'y a pas encore de situation de prise de pouvoir, donc aucun rapport de force réel entre les groupes.

40 Si nous suivons BESNIER (2001) dans l'idée que cette intervention des sages «ne nous porte pas à une vénération exorbitante à l'égard de ceux qui en ont été à l'origine - les législateurs n'ont pas été des dieux, ni des surhommes -, ni à un attachement «religieux aux mesures instituées» (128), nous ne partageons pas le jugement qui consiste à y voir seulement le produit d'une «compréhension assez simple 
Hermarque, ces sages politiques lucrétiens ne sont pas nomothètes; ces hommes plus doués que les autres ne produisent pas une véritable législation, mais ils préparent la communauté à fonder ses propres lois, par la prise de conscience de sa profonde communauté. Ils l'invitent à une forme d'autorégulation qui va varier selon les lieux et les temps; ils ne disent pas ce qui est juste mais quel critère adopter pour évaluer le juste.

Comment interpréter cette différence entre nomothètes et éducateurs? Il nous semble qu'il s'agit là de deux figures possibles de l'intervention politique, non pas contradictoires, mais dialectiques - c'est à dire, imaginées pour résoudre cette tension intrinsèque à l'épicurisme, entre la nécessaire universalité de la salvation philosophique, et l'impossibilité de cette même universalité appliquée à la sécurisation politique. En philosophie, il ne peut y avoir de mieux ou moins doués; mais tout homme est capable de recevoir l'enseignement d'Épicure et de construire ainsi l'espace intime de sa sécurité, au sein duquel il n'y a plus de peur car il n'y a plus d'opinions vides ni de dangers ${ }^{41}$. Le rôle pédagogique du sage est alors très clair: il est de diffuser la doctrine, comme ensemble objectif et constitué d'énoncés rationnels recevables par chaque individu pris individuellement. Il s'agit d'une pédagogie verticale et dogmatique, depuis un maître vers un élève, qui repose sur le principe simple d'une assimilation de la doctrine au sein des lieux privilégiés car intimes de la communauté des philoi; dans le cercle des amis, le maître s'adresse à des personnes autonomes et

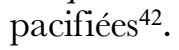

des avantages qu'on en peut retirer (et des limites au-delà desquelles ces avantages risquent de ne plus être obtenus)»; si la compréhension était simple, elle serait partagée spontanément par tous, ce qui n'est pas le cas, et l'absence de vénération religieuse pour la figure du législateur ou du politique ne se fonde pas sur la facilité de son intervention.

${ }^{41}$ Les différents relecteurs de cet article ont remarqué une apparente similitude entre la tripartition des individus que nous distinguons dans l'espace politique, et la distinction attribuée par Sénèque dans la lettre LII à Epicure lui-même entre les différentes aptitudes à la philosophie: le génie autodidacte, qui n'a pas besoin d'enseignement pour trouver seul la vérité, le disciple très doué qui suit aisément son maître, incarné par Métrodore, enfin le disciple plus lent qui a besoin non seulement d'un modèle mais d'une force pour le pousser à bien faire et bien penser («quibus non duce tantum opus sit, sed adiutore et, ut ita dicam, coactore», Sen. Ep. 52, 3), représenté par Hermarque. Mais nous voyons une très grande différence entre les deux situations, philosophique et politique, différence qui repose sur deux éléments importants: d'une part cette dernière catégorie est valorisée et non dépréciée par Sénèque, qui considère que ces élèves moins doués sont en réalité les plus admirables et les plus méritants des apprentis philosophes («tamen maior est laus idem effecisse in difficiliore materia»); d'autre part il n'y aucune différence de nature entre la sagesse détenue ou apprise par ces différents types d'apprentis, il s'agit bien toujours de la même philosophie («ad eundem finem uterque pervenerit») - alors que sur le plan politique, les moins doués n'ont jamais réellement accès à l'intelligence des lois. Il nous paraît donc bien que ce texte de Sénèque renforce l'hypothèse de l'universalité au moins potentielle du salut philosophique, indépendamment des qualités intrinsèques des individus.

42 On pourrait opposer à cette universalité potentielle de la salvation philosophique l'évocation régulière dans le corpus épicurien de la notion de diathesis, disposition individuelle qui conditionnerait la possibilité de la vie philosophique. La diathesis comme disposition est explicitement évoquée par Diogène d'Oenoanda au fragment 111: «Ce n'est pas la nature qui nous a fait nobles ou vils - assurément elle est une pour tous -, mais ce sont nos actions et nos dispositions» (trad. Morel dans DeLATTRE et al. 2010). Cette diathesis peut être interprétée, dans son versant strictement matérialiste, comme une configuration atomique propre à l'individu qui le rendrait plus ou moins apte à assimiler la sagesse, et 
La situation de l'éducateur politique est beaucoup plus difficile: il ne s'adresse pas à l'individu mais à la collectivité, hoi polloi, dans un lieu et un temps donnés, et il doit composer avec les limites de compréhension de certains membres de la collectivité, limites qui sont comme on l'a vu en grande partie causées par la vie sociale elle-même - c'est-à-dire par la situation des individus au sein d'un espace non plus privé mais public. Son rôle est alors double: il est à la fois, comme chez Lucrèce, celui qui va rendre viable la collectivité en l'adaptant à l'idée même d'une vie politique, dans une perspective quasiment transcendantale; et celui qui va plus prosaïquement, comme chez Hermarque, faire usage de ses capacités intellectuelles supérieures pour concevoir les lois elles-mêmes, c'est à dire pour appliquer réellement à une communauté donnée la prénotion du juste via un système législatif.

Or, si la première de ces deux fonctions, métapolitique, pourrait éventuellement être considérée comme philosophique, la seconde ne l'est plus. Elle relève d'une pratique spécifique qui n'a plus réellement à voir avec la science de la nature ellemême, même si elle demande des capacités exceptionnelles. Cette nuance explique peut-être une série de contresens, favorisés par des lectures malveillantes des thèses politiques épicuriennes ${ }^{43}$, qui mettent le désengagement du sage sur le compte d'un mépris général pour la vie collective. La politique n'est pas le métier du philosophe, parce qu'il n'y a pas besoin d'être philosophe pour exercer cette pratique - et que politique et philosophie n'interviennent pas dans le même lieu ${ }^{44}$. Ce qui ne signifie pas que l'activité politique ne suppose pas une intelligence spécifique qui, par certains aspects, sera proche de l'intelligence du philosophe - une capacité à anticiper et à exercer son raisonnement en faisant usage de prénotions correctement construites,

dans son versant plus comportementaliste, comme une disposition susceptible d'orienter les actions et décisions dans l'une ou l'autre direction; les individus dotés d'une mauvaise diathesis seraient alors beaucoup plus difficiles à éduquer que les autres. Cette lecture, qui est notamment celle de GRILLI (1983) reprise par ERLER (2011), peut être considérablement atténuée par la prise en compte de la relation complexe établie par exemple par Philodème dans le De ira entre disposition, émotions et éducation (cf. Asmis 2011), relation qui semble pointer vers une compréhension dynamique et non statique de la disposition: la diathesis est susceptible d'une évolution favorable grâce à l'éducation. Cette dynamique est d'ailleurs nous semble-t-il déjà esquissée dans le fragment 111 cité ci-dessus, du fait de son association forte aux «actions». Nous remercions le relecteur de cet article qui a attiré notre attention sur ces éléments bibliographiques importants pour notre propos.

43 Par exemple chez Cicéron, Plutarque ou Sénèque qui reprennent à de multiples reprises à leur compte le trope de l'épicurien indifférent car désengagé de la vie politique. Cf. là dessus ASMIS (2001), 113, 120. ROSKAM (2007) a livré récemment une analyse exhaustive et magistrale de ce lieu commun philosophique extrêmement vivace.

${ }^{44}$ Voir par exemple Philodème qui, dans la Rhétorique (SudHaus 1895, Suppl. 27, 20 = Us. 10) discute le point de savoir si la rhétorique politique est bien une technè. Elle n'est en tout cas ni une partie de la science de la nature ni un art savant, mais une empeiria; de ce fait, elle ne concerne pas en priorité le philosophe, qui n'est pas le mieux placé pour l'exercer. Voir par exemple BLANK (1996 et 2001). Sur le même thème, Plutarque dans le Contre Colotès rapporte à la fois la vénération pour l'œuvre des premiers législateurs qui ont assuré la tranquillité politique $(30,1124 \mathrm{~d})$, mais aussi les attaques cruelles de Métrodore contre les sages qui, par vanité, ont voulu jouer au politique «en se prenant pour Lycurgue ou Solon» (1127b). Sur cette question chez Lucrèce, voir FowLER (1989). 
afin de limiter le plus possible les difficultés liées à la permanence irréductible de la confusion du juste.

\section{Bibliographie}

Asmis (2001):

Asmis, E., «The politician as public servant in Cicero’s De Re Publica», in Auvray-Assayas, C. and Delattre, D. (éds.), Cicéron et Philodème: la polémique en philosophie ("Études de littérature ancienne" 12), Paris, 109-128.

Asmis (2011):

Asmis, E., «The necessity of anger in Philodemus' On angen», in Fish, J., Sanders, K.R. (eds.), Epicurus and the Epicurean tradition, Cambridge-New York, 152-182.

Austin (2012):

Austin, E.A., «Epicurus and the politics of fearing death», Apeiron 45 (2), 109-129.

Blank (1996):

Blank, D., «Philodemus on the technicity of rhetoric», in Giannantoni, G., Gigante, M. (eds.), Epicureismo greco e romano: atti del congresso internazionale (Napoli, 19-26 maggio 1993) = Elenchos 25, Napoli, 585-596.

Blank (2001):

Blank, D., «La philologie comme arme philosophique: la connaissance technique de la rhétorique dans l'épicurisme», in Auvray-Assayas, C., Delattre, D. (éds.), Cicéron et Philodème: la polémique en philosophie ("Études de littérature ancienne” 12), Paris, 241-257.

Besnier (2001):

Besnier, B., «Justice et utilité de la politique dans l'épicurisme: réponse à Élizabeth Asmis», in Auvray-Assayas, C., Delattre, D. (éds.), Cicéron et Philodème: la polémique en philosophie ("Études de littérature ancienne" 12), Paris, 129-157.

Bouffartigue (1977):

Porphyre, De l'abstinence, I, Livre I, ed. J. Bouffartigue et M. Patillon, (“Collection Guillaume Budé”), Paris.

Delattre (2010):

Delattre, D. et al. (eds.), Les Épicuriens ("Bibliothèque de la Pléiade” 564), Paris.

Erler (2011):

Erler, M., «Autodidact and student: on the relationship of authority and autonomy in Epicurus and the Epicurean tradition», in Fish, J., Sanders, K.R. (eds.), Epicurus and the Epicurean tradition, Cambridge-New York, 9-28.

Essler (2011):

Essler, H., Glückselig und unsterblich: epikureische Theologie bei Cicero und Philodem: mit einer Edition von Pherc. 152/157, Kol. 8-10 (“Schwabe Epicurea” 2), Basel.

Fowler (1989):

Fowler, D.P., «Lucretius and politics», in Griffin, M., Barnes, J. (eds.), Philosophia togata: [1] Essays on Philosophy and Roman Society, Oxford, 120-150.

Gernet (1928): 
Gernet, L., «Frairies antiques», Revue des Études Grecques XLI, 313-359.

Giovacchini (2011):

Giovacchini, J. et al., «La philosophie populaire de Polystrate l'épicurien», in Counet, J.M. (éd.), Philosophie et langage ordinaire, Louvain-la-Neuve, 57-82.

Giovacchini (2012):

Giovacchini, J., L'empirisme d'Épicure (“Les Anciens et les Modernes - Études de philosophie" 11), Paris.

Glidden (1985):

Glidden, D.K., «Epicurean prolēpsis», Oxford Studies in Ancient Philosophy III, 175-217.

Goldschmidt (1977):

Goldschmidt, V., La doctrine d'Épicure et le droit ("Bibliothèque d'histoire de la philosophie" 17), Paris.

Grilli (1983):

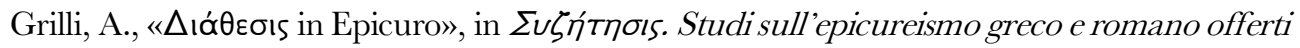
a Marcello Gigante ("Biblioteca della Parola del Passato” XVI), Napoli, 93-109.

Helmer (2017):

Helmer, É., «Aux frontières de la cité: les incurables de Platon», Philosophie Antique 17, 125148.

Kany-Turpin (2016):

Cicéron, Fins des biens et des maux, trad. J. Kany-Turpin, Paris.

Longo Auricchio (1988):

Ermarco, Frammenti, ed., trad. e commento a c. di F. Longo Auricchio ("La scuola di Epicuro" 6), Napoli.

Marcovich (1999):

Diogenis Laertii Vitae philosophorum, ed. M. Marcovich ("Bibliotheca Scriptorum Graecorum et Romanorum Teubneriana"), Stuttgart.

Martin (1969):

Lucretius, De rerum natura, ed. J. Martin (“Bibliotheca Scriptorum Graecorum et Romanorum Teubneriana"), Leipzig.

Mitsis (2014):

Mitsis, Ph., L'éthique d'Épicure: les plaisirs de l'invulnérabilité, trad. par Gigandet, A. (“Les Anciens et les Modernes - Études de philosophie” 21), Paris.

Morel (2000):

Morel, P.-M., «Épicure, l'histoire et le droit», Revue des Études Anciennes 102 (3-4), 393411.

Morel (2008):

Morel, P.-M., «Method and evidence: on Epicurean preconception», in Cleary, J.J., Gurtler, G.M. (eds.), Proceedings of the Boston Area Colloquium in Ancient Philosophy, 23, 2007, Leiden-Boston (Mass.), 25-56.

Morel (2016):

Morel, P.-M., «Travail et émancipation dans l'épicurisme antique: Prométhée revisité», Revue Internationale de Philosophie 278 (4), 451-167. 
Obbink (1988):

Obbink, D.D., «Hermarchus, Against Empedocles», The Classical Quarterly XXXVIII, 428435.

Polansky-Kuczewski (1990):

Polansky, R.M., Kuczewski, M., «Speech and thought, symbol and likeness: Aristotle's De interpretatione 16a 3-9», Apeiron 23 (1), 51-63.

Robitzsch (2017):

Robitzsch, J.M., «The Epicureans on Human Nature and its Social and Political Consequences», Polis. The Journal for Ancient Greek Political Thought 34 (1), 1-19.

Roskam (2007):

Roskam, G., Live unnoticed on the vicissitudes of an Epicurean doctrine ("Philosophia antiqua: a series of studies on ancient philosophy" 111), Leiden-Boston.

Schiche (1915):

M. Tulli Ciceronis Scripta quae manserunt omnia: 43, De finibus bonorum et malorum, ed. Schiche, Th. ("Bibliotheca Scriptorum Graecorum et Romanorum Teubneriana”), Stuttgart.

Schofield (1996):

Schofield, M., «Epilogismos: an appraisal», in Frede, M., Striker, G. (eds.), Rationality in Greek thought, Oxford-New York, 221-237.

Schrijvers (1996):

Schrijvers, P.H., «Lucretius on the origin and development of political life (De rerum natura 5.1105-1160)», in Algra, K.A., van der Horst, P.W., Runia, D.T. (eds.), Polyhistor: studies in the history and historiography of ancient philosophy presented to Jaap Mansfeld on his sixtieth birthday, Leiden, 220-230.

Smith (1993):

Diogenes of Oenoanda, The Epicurean inscription, ed. Ferguson Smith, M. ("La scuola di Epicuro" Supplemento 1), Napoli.

Sudhaus (1895):

Philodemi volumina rhetorica. Supplementum, ed. S. Sudhaus ("Bibliotheca scriptorum graecorum et romanorum Teubneriana"), Lipsiae.

Tsouna (2016):

Tsouna, V., «Epicurean Preconceptions», Phronesis, 61 (2), 160-221.

Vander Waerdt (1987):

Vander Waerdt, P.A., «The Justice of the Epicurean Wise Man», The Classical Quarterly 37 (2), 402-422.

Vander Waerdt (1988):

Vander Waerdt, P.A., «Hermarchus and the Epicurean Genealogy of Morals», Transactions of the American Philological Association (1974-2014) 118, 87-106.

Warren (2009):

Warren, J., «Removing fear», in Warren, J. (ed.), The Cambridge companion to Epicureanism, Cambridge-New York, 234-248. 